\title{
Solitary potential structures observed in the magnetosheath by the Cluster spacecraft
}

\author{
J. S. Pickett ${ }^{1}$, J. D. Menietti ${ }^{1}$, D. A. Gurnett ${ }^{1}$, B. Tsurutani ${ }^{2}$, P. M. Kintner ${ }^{3}$, E. Klatt ${ }^{3}$, and A. Balogh ${ }^{4}$ \\ ${ }^{1}$ Department of Physics and Astronomy, The University of Iowa, Iowa City, IA, USA \\ ${ }^{2}$ Jet Propulsion Laboratory, California Institute of Technology, Pasadena, CA, USA \\ ${ }^{3}$ School of Electrical Engineering, Cornell University, Ithaca, NY, USA \\ ${ }^{4}$ The Blackett Laboratory, Imperial College, London, UK
}

Received: 2 January 2002 - Revised: 16 April 2002 - Accepted: 17 April 2002

\begin{abstract}
Bipolar pulses of $\sim 25-100 \mu$ s in duration have been observed in the wave electric field data obtained by the Wideband plasma wave instrument on the Cluster spacecraft in the dayside magnetosheath. These pulses are similar in almost all respects to those observed on several spacecraft over the last few years. They represent solitary potential structures, and in this case, electron phase space holes. When the time series data containing the bipolar pulses on Cluster are transformed to the frequency domain by a windowed FFT, the pulses appear as typical broad-band features, extending from the low-frequency cutoff of the bandpass filter, $\sim 1 \mathrm{kHz}$, up to as great as $20-40 \mathrm{kHz}$ in some cases, with decreasing intensity as the frequency increases. The upper frequency cutoff of the broad band is an indication of the individual pulse durations (1/f). The solitary potential structures are detected when the local magnetic field is contained primarily in the spin plane, indicating that they propagate along the magnetic field. Their frequency extent and intensity seem to increase as the angle between the directions of the magnetic field and the plasma flow decreases from $90^{\circ}$. Of major significance is the finding that the overall profile of the broad-band features observed simultaneously by two Cluster spacecraft, separated by a distance of over $750 \mathrm{~km}$, are strikingly similar in terms of onset times, frequency extent, intensity, and termination. This implies that the generation region of the solitary potential structures observed in the magnetosheath near the bow shock is very large and may be located at or near the bow shock, or be connected with the bow shock in some way.
\end{abstract}

\section{Introduction}

Broad-band electrostatic noise (BEN) was first identified by Scarf et al. (1974) and Gurnett et al. (1976) from measurements obtained with a spectrum analyzer in the distant tail. BEN was characterized as bursty and consisting of broad-

Correspondence to: J. S. Pickett (jolene-pickett@uiowa.edu) band spectral features usually extending from the lowest frequencies measured, up to as high as the plasma frequency, with decreasing intensity as the frequency increases. After this discovery, BEN was observed in several other regions of the Earth with various spacecraft, including the magnetosheath (e.g. Rodriguez; 1979; Anderson et al., 1982).

In 1994, Matsumoto et al. (1994) made one of the most important new discoveries of the decade with respect to BEN. Upon examining the Geotail PWI time series data, they found that solitary waves of a few milliseconds in duration were responsible for the high frequency part of BEN in the geomagnetic tail as the spacecraft crossed the plasma sheet boundary layer. This brought renewed attention to previous observations of solitary waves, also described as solitary bipolar pulses, by S3-3 (Temerin et al., 1982) and Viking (Koskinen et al., 1987), as well as a heightened anticipation of the high time resolution waveform measurements being made on Polar and FAST in the mid to late 1990s. Kojima et al. (1999a) have presented some of the characteristics of these solitary waves in the various regions of space. They discuss the similarities and differences in terms of pulse width, amplitude, flow direction to the magnetic field and flow speed.

By 1998, these pulses were found to be indicative of coherent potential structures (Franz et al., 1998), with the initial sign of the pulse (positive or negative) determining whether the structure was an electron or ion phase space hole. An interferometer on Polar was used to make these measurements. Ergun et al. (1998) found that solitary structures detected by FAST in the auroral acceleration region, called "fast solitary waves", propagated along the magnetic field at speeds of a few thousands $\mathrm{km} / \mathrm{s}$ and had amplitudes up to $2.5 \mathrm{~V} / \mathrm{m}$.

With the discovery that BEN consists of a series of solitary waves rather than being broad banded, several researchers began performing simulations using experimental data as input, in order to explore generation mechanisms. Some of the most important results to come out of these simulations are that electron phase space holes can be generated by several mechanisms. One involves counter-streaming cold electron beams (Goldman et al., 1999) with electrostatic whistlers and 
lower hybrid waves as an end product. Another involves double layers, or strong potential ramps (Singh, 2000; Newman et al., 2001), which accelerate an electron beam and thus, produce electron phase space holes by a spatial two-stream instability. Yet another mechanism involves the nonlinear evolution of the electron beam instability leading to the formation of Bernstein-Greene-Kruskal (BGK) potential structures (Omura et al., 1996; Muschietti et al., 1999). Most of these simulations have used the FAST and Geotail data from the auroral acceleration region and geomagnetic tail, respectively, as their input, such that it is unknown at this time whether these simulations are pertinent in other regions of space, such as in the magnetosheath. Kojima et al. (1999a, 1999 b) suggest the existence of a common generation mechanism related to electron dynamics based on a comparison of the time scales of the solitary waves observed in the magnetosheath to those in the magnetosphere and in the geomagnetic tail with Geotail, FAST and Polar data.

The present study of bipolar pulses makes use of the availability for the first time of identical high time resolution waveform measurements obtained simultaneously on two spacecraft located in the same general region of the dayside magnetosheath near the bow shock. We first describe the waveform receiver and magnetometer that were used to make the Cluster measurements, followed by a presentation of the observations made by the waveform receiver. We continue with an overview of supporting data, particularly the magnetometer data, that are useful in helping us to understand the wave measurements. We conclude with a discussion of the significance of the observations and a summary of the results.

\section{Instrumentation}

A Wideband (WBD) plasma wave receiver is mounted on each of the four Cluster spacecraft, which are in an approximately $4 \times 20 R_{E}$ polar orbit around the Earth. The wideband technique involves transmitting band-limited waveforms in real time directly to a Deep Space Network ground station using a high-rate data link (Gurnett et al., 1997). WBD processes signals from one of four antennas, measuring $\boldsymbol{E}$-fields via either of two dipole, spherical probe antennas located in the spin plane, or $\boldsymbol{B}$-fields via magnetic search coils located either in the spin plane or along the spin axis. The distance between the two electric field spheres is approximately $90 \mathrm{~m}$.

The WBD instrument has three band-pass filter ranges available: $1 \mathrm{kHz}$ to $77 \mathrm{kHz}, 50 \mathrm{~Hz}$ to $19 \mathrm{kHz}$, and $50 \mathrm{~Hz}$ to $9.5 \mathrm{kHz}$. In this paper, we will be presenting data obtained while the instrument was configured with the $1 \mathrm{kHz}$ to $77 \mathrm{kHz}$ band-pass filter, 8 bits/sample resolution, and the electric $E_{z}$ (spin plane) antenna selected as the sensor input. In this mode the instrument has a duty cycle of $12.5 \%$ and a sample rate of $219.5 \mathrm{kHz}$. Duty cycling is required because the bit rate exceeds the spacecraft telemetry rate of $220 \mathrm{kbit} / \mathrm{s}$. The WBD gain is automatically updated according to predetermined criteria in $5 \mathrm{~dB}$ steps (a total of 16 possible steps)

\section{CLUSTER ORBIT LOCATION/CONFIGURATION May 8, 2001 at 23:00 UT}

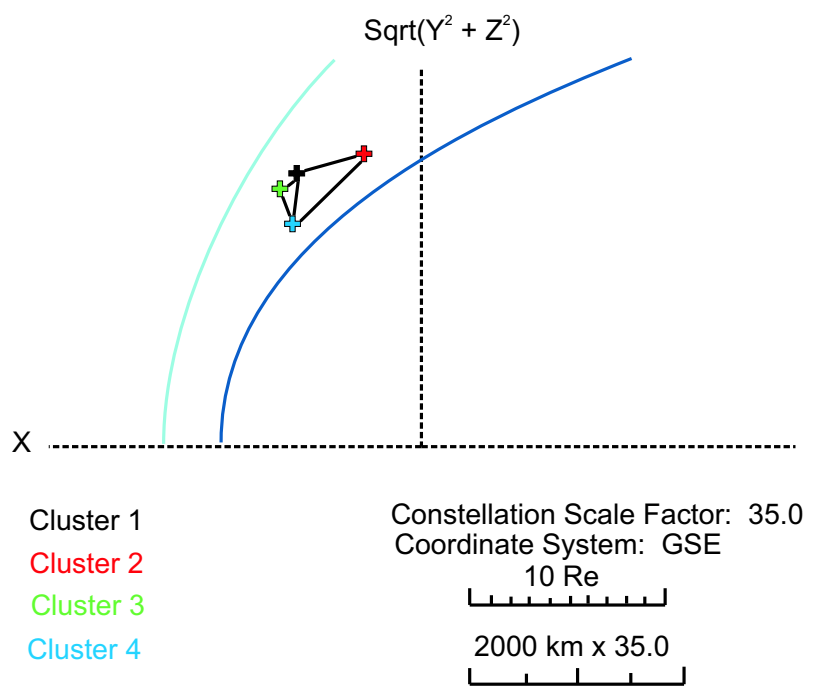

Fig. 1. Locations of the Cluster spacecraft on 8 May 2001 at 23:00 UT and the relative positions of the spacecraft with respect to the nominal bow shock (light blue curve) and magnetopause (dark blue curve). Note that the scale of the spacecraft separations has been expanded 35 times in order to see the details of the separations. Spacecraft 3 (green cross) is the reference spacecraft located at the true position of the Cluster quartet. The data presented in Fig. 2 are from Cluster 1 (black cross) and Cluster 3 (green cross).

over a preselected rate ranging from 0.1 to $27 \mathrm{~s}$ in increments of $0.1 \mathrm{~s}$. The update rate for all of the data presented in this paper is $1 \mathrm{~s}$. For further details on the Cluster WBD instrument, refer to Gurnett et al. (1997).

The magnetic field investigation (FGM) on Cluster provides accurate, high time-resolution, intercalibrated measurements of the magnetic field vector along the Cluster orbit (Balogh et al., 1997). Each Cluster spacecraft carries an identical instrument consisting of two triaxial fluxgate magnetometers and an onboard data-processing unit. In order to minimize the magnetic background of the spacecraft, one of the magnetometer sensors is located at the end of a $5.2 \mathrm{~m}$ radial boom, and the other is located at $1.5 \mathrm{~m}$ inbound from that location. Selection of one of these as the primary sensor is made by ground command. For further information on the Cluster FGM instrument, see Balogh et al. (1997).

\section{Experimental observations}

The data presented in this paper were obtained in the dayside magnetosheath at $15.6-15.8 R_{E}$. The Cluster spacecraft were outbound and about 1.2-1.4 $R_{E}$ earthward of the nominal bow shock (see Fig. 1). They were located around $+17^{\circ}$ magnetic latitude and 7.8 Magnetic Local Time. WBD data from spacecraft 1 and 3 were obtained simultaneously during this period and are shown in the spectrograms of Fig. 2. The data were obtained on 8 May 2001 from 22:49 to 23:10 UT. 
Cluster WBD Plasma Wave Data, Ez Antenna

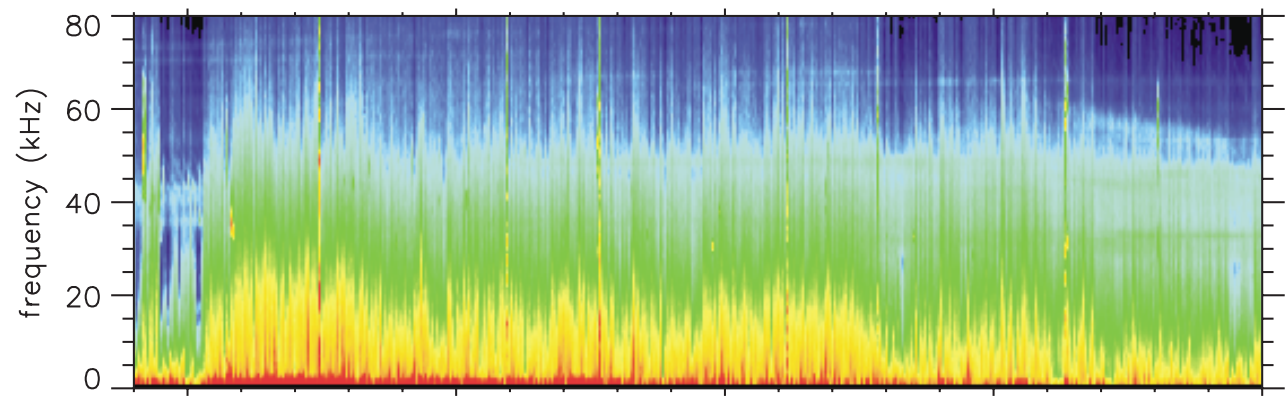

$$
80
$$

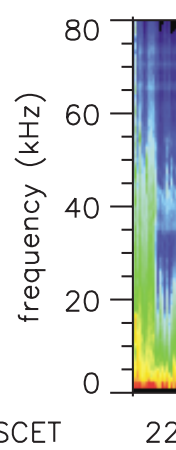

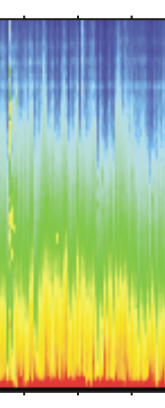

$$
\text { scer }
$$

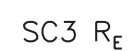

scos mot

SC3 MLT

SC3 L
22:50

15.64

17.27

7.80

17.20

2001-05-08
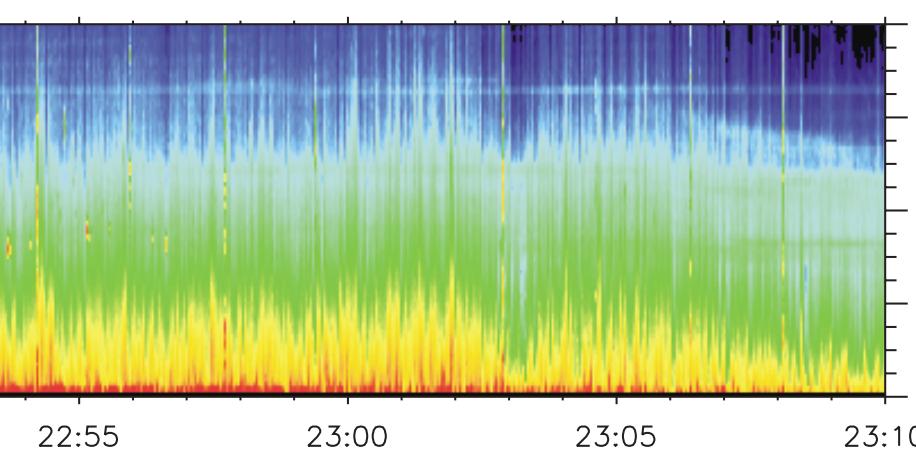

\section{$22: 55$}

15.69

16.97

7.80

17.10

(128) 22:49:00

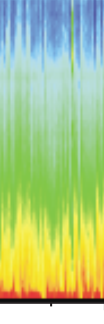

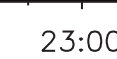

15.74

16.66

7.81

17.10
SCET
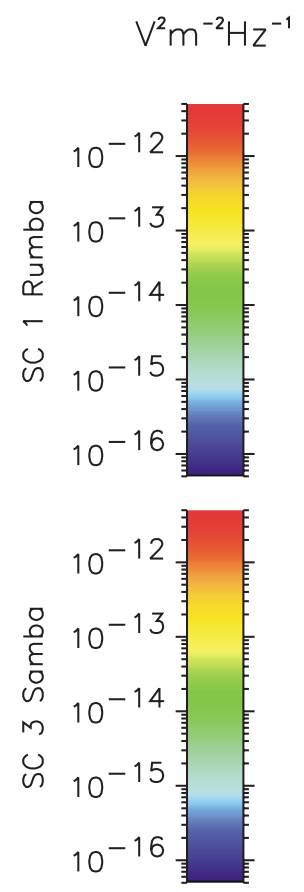

Fig. 2. Cluster WBD spectrograms from spacecraft 1 and 3 obtained on 8 May 2001 while in the dayside magnetosheath. These spectrograms show a similar profile of broad-band features (onset, frequency extent, intensity, termination) on both spacecraft, yet the spacecraft are separated by about $750 \mathrm{~km}$.

Data from spacecraft 1 are plotted in the top panel and from spacecraft 3 in the bottom panel (WBD was not telemetering data from spacecraft 2 and 4 at this time). In Fig. 2, frequency is plotted on the vertical axis vs. time on the horizontal axis, with colour representing power spectral density. Orbit parameters for spacecraft 3 are provided in columnar format beneath the spectrograms. During this period of time, the electron plasma frequency was $30 \mathrm{kHz}$ or greater and the electron cyclotron frequency was approximately $1 \mathrm{kHz}$ (the basis for the values of these parameters will be briefly discussed in Sect. 4). The interference line seen on both spacecraft, but more strongly on spacecraft 3 , at about $65 \mathrm{kHz}$, is associated with the spacecraft batteries. The spectrograms were created on the ground by applying a windowed Fast Fourier Transform (WFT) to the time series.

Most striking in Fig. 2 are the broad-band features observed on both spacecraft extending from around $1 \mathrm{kHz}$ up to around $20-40 \mathrm{kHz}$. The overall profile of these broad-band features (onset, frequency extent, intensity, termination) is exceptionally similar on both spacecraft for this period of time. What makes this observation so unexpected is that the spacecraft are separated by approximately $750 \mathrm{~km}$ at this time.
In order to understand the emission that is observed in Fig. 2, it is necessary to look at the waveforms that were used to create the spectrograms. A sample of the calibrated WBD time series from each of the spacecraft is plotted in Figs. 3a (spacecraft 1) and 4a (spacecraft 3 ). These plots, each covering approximately $4.7 \mathrm{~ms}$, provide the electric field magnitude, in $\mathrm{mV} / \mathrm{m}$, on the vertical axis vs. the elapsed time since the start time shown at the top of the figure, in seconds, on the horizontal axis.

The prominent waveforms seen in Figs. 3a and 4a, e.g. Fig. 4a at $0.0014 \mathrm{~s}$, are what are commonly referred to as bipolar pulses. These are waveform signatures in which a significant, isolated pulse over background is observed, consisting of a half sinusoid-like cycle, followed by a similar half cycle having opposite sign. Several of these bipolar pulses are observed in Figs. 3a and 4a on each spacecraft with typical time durations of approximately $70 \mu$ s and peak-to-peak fields of $0.1-0.5 \mathrm{mV} / \mathrm{m}$. With respect to the initial direction of the pulses (positive or negative), we see both directions with no clear preference on either spacecraft. Since WBD uses the two spheres from one antenna only in a dipole configuration rather than monopole, there is no conclusive way for us to determine whether the solitary potential structures 
CLUSTER 1 WAVELET ANALYSIS

May 8, 2001, Start Time 22:50:20.488 UT

a) Cluster WBD Time Series

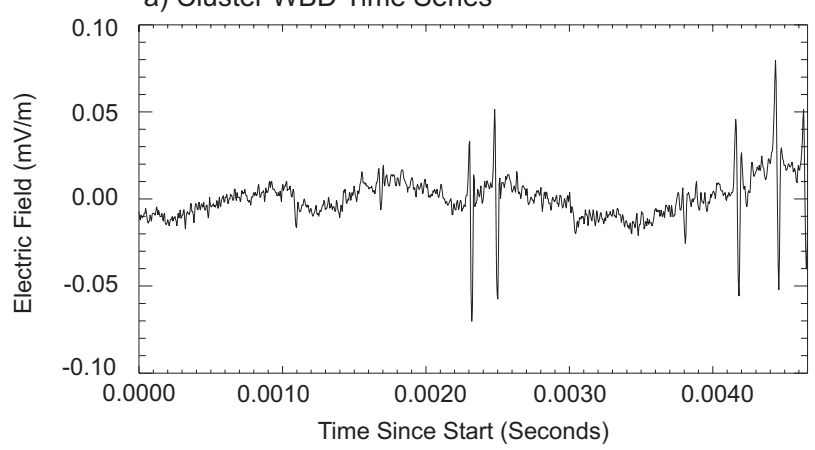

b) Wavelet Power Spectrum

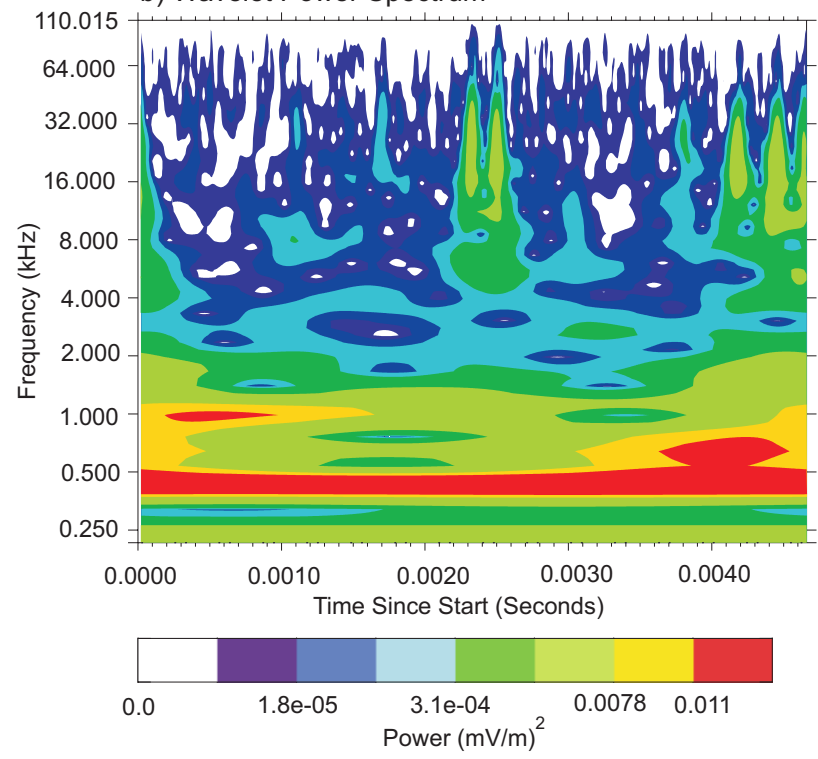

Fig. 3. (a) WBD time series data for a $4.7 \mathrm{~ms}$ time interval from Cluster 1 during the time period shown in Fig. 2. Solitary bipolar pulses are observed along with a low-frequency sinusoidal waveform. The pulses are what creates the broad bands in Fig. 2. (b) Shows the representation of these pulses in frequency and time using a wavelet transform. In this representation, the solitary bipolar pulses are not broad banded.

are positive (implying electron phase space holes) or negative (implying ion phase space holes). However, numerous research on this topic, both experimental and theoretical, would suggest that due to the shortness of the pulses, the solitary potential structures being observed by the Cluster WBD in the magnetosheath must be electron phase space holes. For example, using the criteria of Kojima et al. (1999a, 199b), we find that a typical duration of a pulse in the magnetosheath, $70 \mu \mathrm{s}$, multiplied by the plasma frequency, $30 \mathrm{kHz}$, gives a characteristic time scale of 2.1 for these solitary waves. This is comparable to the Geotail, Polar and FAST results (values between 1 and 10), implying that these structures are closely
CLUSTER 3 WAVELET ANALYSIS

May 8, 2001, Start Time 22:50:19.532 UT

a) Cluster WBD Time Series

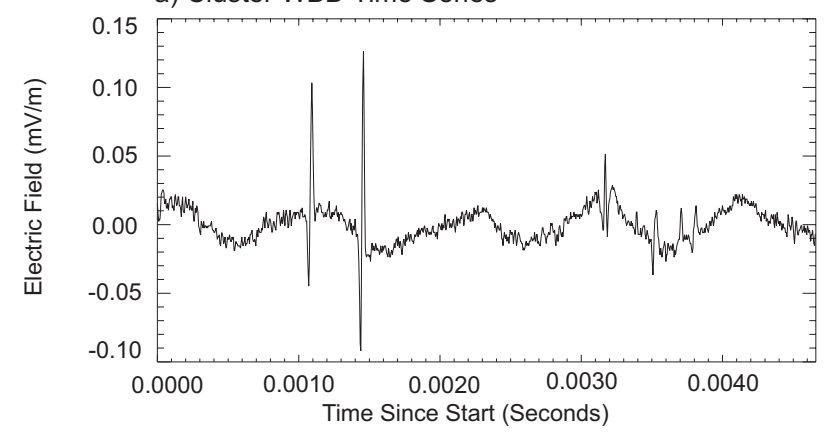

b) Wavelet Power Spectrum

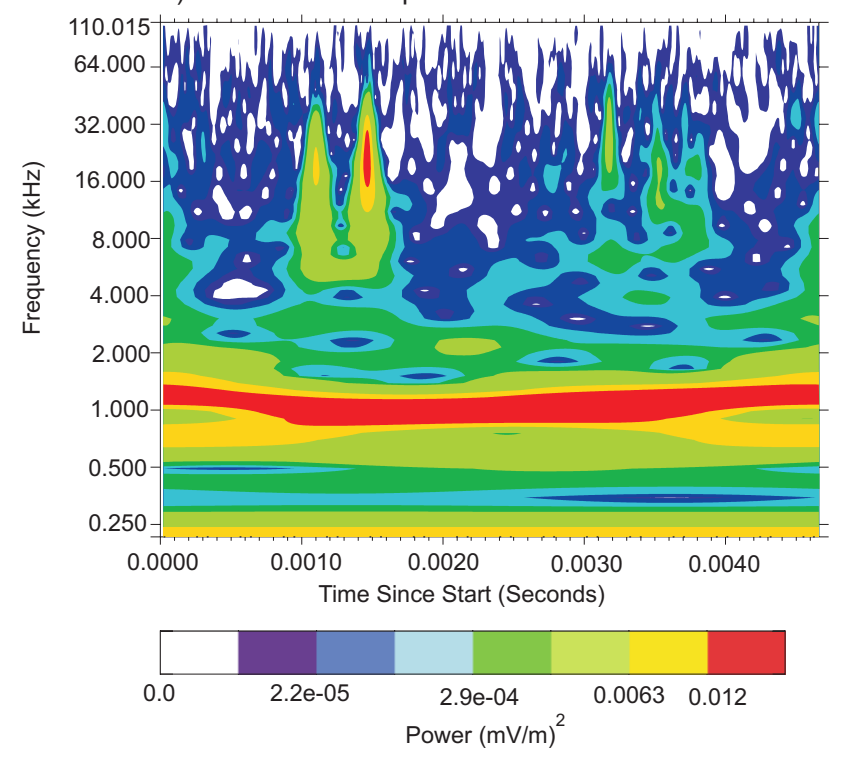

Fig. 4. (a) WBD time series data for a $4.7 \mathrm{~ms}$ time interval from Cluster 3 during the time period shown in Fig. 2. Solitary bipolar pulses are observed along with a low-frequency sinusoidal waveform. The pulses are what creates the broad bands in Fig. 2. (b) Shows the representation of these pulses in frequency and time using a wavelet transform. In this representation, the solitary bipolar pulses are not broad banded.

related to electron dynamics, since the pulse widths are on the order of the electron plasma oscillation periods.

The bipolar pulses with time durations typically ranging from 25 to $100 \mu$ s are observed throughout the entire time interval plotted in Fig. 2, with the exception of where the dark blue background (approximately $10^{-16} \mathrm{~V}^{2} \mathrm{~m}^{-2} \mathrm{~Hz}^{-1}$ ) is observed to extend down to frequencies below about $5 \mathrm{kHz}$, e.g. around 22:49:30, 22:50:10, and 23:03:00 UT. The bipolar pulses are represented in the spectrogram as the broad bands which extend from $1 \mathrm{kHz}$ (the lower edge of the filter) up to as great as $20-40 \mathrm{kHz}$, with power spectral density decreasing as the frequency increases. The WFT used in 
Fig. 2 is an inaccurate method for obtaining time-frequency localization, since it imposes a scale or "response interval" $T$ into the analysis. The inaccuracy arises from the aliasing of high- and low-frequency components that do not fall within the frequency range of the window (Torrence and Compo, 1998). Thus, the bipolar pulses are more accurately represented in the frequency domain when a wavelet transform using a Morlet wavelet is applied to the times series, as shown in Figs. $3 b$ and $4 b$, since the wavelet transform provides a time-frequency localization that is scale independent. The Morlet wavelet consists of a plane wave modulated by a Gaussian and best approximates the pulses we are trying to analyze. With the wavelet transform we are better able to see what the pulse looks like in time and frequency. We no longer see the broad band, but rather see the peak power associated with the characteristic frequency (time duration, or $1 / \mathrm{f}$ ) of the pulse as it passes by the spacecraft.

Although the WFT tends to misrepresent the bipolar pulses as broad-band emissions, it does give us valuable insight into the longer term, larger picture of what is going on in this region of space (since the benefits of a wavelet transform would be lost over such a long time interval). For example, as pointed out earlier, the overall frequency-intensity profile is very similar between the two spacecraft. The onset of the bipolar pulses around 22:50:15 UT occurs almost simultaneously on both spacecraft. Looking at the high-time resolution waveforms, we detect the onset to be approximately $0.9 \mathrm{~s}$ earlier on spacecraft 3 than on spacecraft 1 . If we take this delay time as typical from one spacecraft to another and compare the respective time series from both spacecraft based on this delay, we find that there appears to be no correlation with respect to the individual pulses observed on both spacecraft. Referring to Figs. $3 \mathrm{a}$ and $4 \mathrm{a}$, we see that the start time of the time series from spacecraft 3 is about $0.9 \mathrm{~s}$ earlier than that of spacecraft 1 . While it is tempting for the eye to correlate the first two pulses on both spacecraft, as well as the latter group of four, the amplitudes and time between pulses vary too much from one spacecraft to another for us to conclude that the two spacecraft are viewing the same structures represented by the pulses.

Finally, and most important, is that the frequency extent of the broad bands shown in Fig. 2 give an indication of the duration of the individual pulses. For example, the broad bands seen extending up to $40 \mathrm{kHz}$ are indicative of pulses that occur on time scales of $25 \mu \mathrm{s}$. Only the WBD instrument on Cluster is capable of resolving these fast pulses in the time domain due to a $\Delta t$ of $4.5 \mu \mathrm{s}$ between samples in this mode. Other instruments which detect broad bands in the frequency domain, but which do not provide the waveforms, provide inconclusive evidence of these bipolar pulses. This is because broad bands similar to those observed in Fig. 2 can also be indicative of electrostatic turbulence when an FFT-type algorithm has been used to process the time series. Thus, the waveforms are absolutely essential in determining whether the broad bands represent bipolar pulses or some other signature of the wave electric field.

Some of the other emissions observed in Fig. 2 are oc- casional bursts of Langmuir waves at the plasma frequency, e.g. at 22:53:42 UT, as well as very weak escaping continuum seen above the broad bands, and a very intense low-frequency emission below about $3 \mathrm{kHz}$. The continuum is obviously not related to the bipolar pulses, since it is propagating above the plasma frequency. Not so clear is the connection, if any, of the low-frequency emission. These low-frequency waves are obvious in the waveforms of Figs. $3 \mathrm{a}$ and $4 \mathrm{a}$ (the sinusoidal waveforms with a field of about $0.04 \mathrm{mV} / \mathrm{m}$, peak-to-peak), and in the spectra of Figs. $3 \mathrm{~b}$ and $4 \mathrm{~b}$ around $500-1500 \mathrm{~Hz}$. Since the filter rolls off steeply below $1000 \mathrm{~Hz}$, we cannot trust the calibration of any emission seen below that frequency. However, the emission observed in Fig. $4 \mathrm{~b}$ around $1000-1500 \mathrm{~Hz}$ can be trusted. We believe that these low-frequency waves are ion acoustic waves based on their falling in the range $f_{\pi} \leq$ $f \leq f_{p e}$. They are also quite similar to the low-frequency Doppler-shifted ion acoustic wave packets observed in the solar wind at the L1 point by the WIND spacecraft (Mangeney et al., 1999). We point out that the examples of these low-frequency sinusoidal waves shown in Figs. 3 and 4 are not typical of the intensities of these waves seen throughout Fig. 2. We specifically chose these examples in order to highlight the bipolar pulses.

\section{Supporting data}

We have examined the DC magnetic field data from the FGM experiment on Cluster for the time period shown in Fig. 2. Panel (a) of Fig. 5 contains the magnitude of the magnetic field for spacecraft 1 (dashed line) and spacecraft 3 (solid line) for a time period that encompasses that of Fig. 2. Panel (b) contains the magnitude of the component of that magnetic field that lies within the spin plane of each spacecraft for the same time period. Panels (c) and (d) provide the cone angle for spacecraft 1 and 3, respectively, calculated as the angle between the direction of the magnetic field as measured by FGM and the plasma flow, which we assume is along the $X$-axis in a GSE coordinate system. No particle data are available from Cluster for this period of time from which to determine the flow direction, since maneuvers had occurred only a few hours before and the particle instruments were put in a saving mode. From Fig. 5, we deduce that the rapid increase in the magnitude of the magnetic field starting at about 22:50:15 UT is associated almost entirely with the component of the field that is in the spin plane. Thus, it is clear that WBD detects the bipolar pulses during the time that the local magnetic field lies almost entirely within the spin plane of the spacecraft, which is the plane in which WBD senses. Further, the overall profile of the bipolar pulses, as represented by the broad bands in Fig. 2, very closely approximates the profile of the component of the magnetic field contained in the spin plane. The magnetic field data also show that spacecraft 3 observes the change to a spin-plane dominated direction first, which is consistent with the WBD data. Further, from panels 5(c) and 5(d), it appears as if there is 


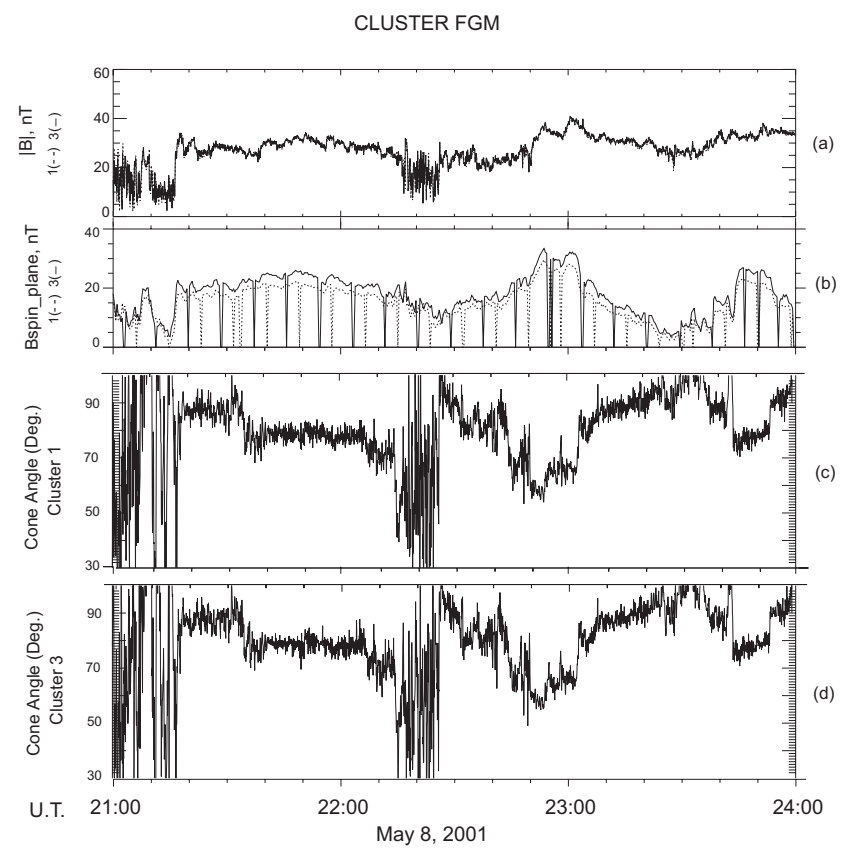

Fig. 5. (a) FGM total magnetic field data from Cluster 1 (dashed line) and Cluster 3 (solid line). (b) The component of the magnetic field that lies in the spin plane of the spacecraft for each of Cluster 1 and Cluster 3. (c) and (d) The cone angle, which is the angle between the direction of the magnetic field and the direction of the plasma flow (assumed to be along the GSE $X$ direction), for Cluster 1 and Cluster 3, respectively.

a dependence on the cone angle, i.e. the broad-band features (solitary structures) decrease in intensity and frequency extent (meaning that $\Delta t$ of the bipolar pulses has increased) or go away, and the low-frequency sinusoidal waves decrease in intensity or go away the closer this angle gets to $90^{\circ}$.

To see whether both spacecraft 1 and 3 lie along the same magnetic field line, we have transformed the position of the spacecraft into a local magnetic field-aligned coordinate system using the FGM data, as shown in Fig. 6. The $\Delta z$ axis is parallel to the magnetic field. The $\Delta y$ axis is in the $\boldsymbol{B} \times \boldsymbol{R}$ direction, where $R$ is the radius vector from the Earth to the center of mass of the 4 Cluster spacecraft, and $\Delta x$ completes the right-handed system. The positions of spacecraft 1 and 3 relative to the center of mass of the four spacecraft in the local magnetic field-aligned system are shown in Fig. 6. We find that the spacecraft do not lie along the same magnetic field line, but rather lie on different field lines separated by approximately $650 \mathrm{~km}$.

In order to put some of the wave observations into perspective, we note that the electron cyclotron frequency varies from about 840 to $1120 \mathrm{~Hz}$ during the time period of Fig. 2 based on FGM data. The electron plasma frequency is $\geq 30 \mathrm{kHz}$ based on occasional bursts of Langmuir waves and on the lower frequency cutoff of the continuum that is observed during periods of low or absent bipolar pulses. The $\mathrm{Kp}$ index is 4 for the three-hour period of 21:00 to 24:00 UT on 8 May 2001. Finally, the solar wind pressure is around 1-
$2 \mathrm{nPa}$, the solar wind speed is close to $500 \mathrm{~km} / \mathrm{s}$, the plasma beta is 0.6 , and IMF $B_{z}$ ranges from -1 to $-6 \mathrm{nT}$ based on ACE data from the L1 point.

\section{Discussion}

The bipolar pulses that are seen throughout the time interval presented in Fig. 2 on 8 May 2001 are consistent with solitary potential structures, in this case electron phase space holes. They are similar in form to the structures observed in the magnetotail and magnetosheath by Geotail, in the auroral acceleration region by FAST, and in the high altitude magnetosphere, cusp, and near-Earth plasma sheet by Polar, as discussed in the Introduction. Since WBD does not have the capability of configuring itself as an interferometer on any one spacecraft, we are unable to determine the velocity, and thus, the scale size of the structures. Even though the series of solitary pulses observed in Figs. 3 and 4 appear to be correlated, the time delay between comparable pulses on each spacecraft are not consistent, and the spacecraft do not lie on the same magnetic field line as seen in Fig. 6. Thus, we conclude that we are not detecting the same bipolar pulses on both spacecraft and we cannot use the two spacecraft as an interferometer to determine velocity and scale size.

With the use of the FGM data, we are able to determine that throughout most of the interval shown in Fig. 2, the magnetic field is located primarily in the spin plane, as shown in Fig. 5. It is during these times that WBD detects the structures. This is significant because WBD senses only in the spin plane. The structures are, therefore, confined to the plane containing the magnetic field direction.

We have also examined the cone angle, i.e. the angle between the plasma flow direction and the magnetic field, by using FGM data and by assuming that the plasma flow is generally along the $-X_{\mathrm{GSE}}$ direction. For the interval shown in Fig. 2, we found that this angle is generally between 60 and $90^{\circ}$. As this angle approaches $90^{\circ}$, the broad bands associated with the solitary structures decrease in frequency extent and intensity, and the low-frequency waves decrease in intensity. Any deviation of the plasma flow from the $-X$ direction is expected to be small, but could result in small changes to the cone angle calculations shown in Fig. 5. It appears that the Cluster WBD data support, to some extent, the findings of Coroniti et al. (1994) that the occurrence of the plasma waves from several hundred $\mathrm{Hz}$ to $5 \mathrm{kHz}$ observed by ISEE3 in the distant magnetosheath are nearly absent when the cone angle is large. However, it is not clear from their data whether the broad-band waves are a combination of bipolar pulses and low-frequency sinusoidal waves, as in the case of Cluster, or just one of these, since waveform data were not available to them.

Based on the detection of the structures only when the magnetic field lies primarily in the spin plane, we conclude that the structures are propagating along the magnetic field. Since changes in the magnetic field in the dayside magnetosheath are usually related to changes in the IMF, we be- 
CLUSTER RELATIVE POSITIONS

MAY 8, 2001, 22:50:00 UT
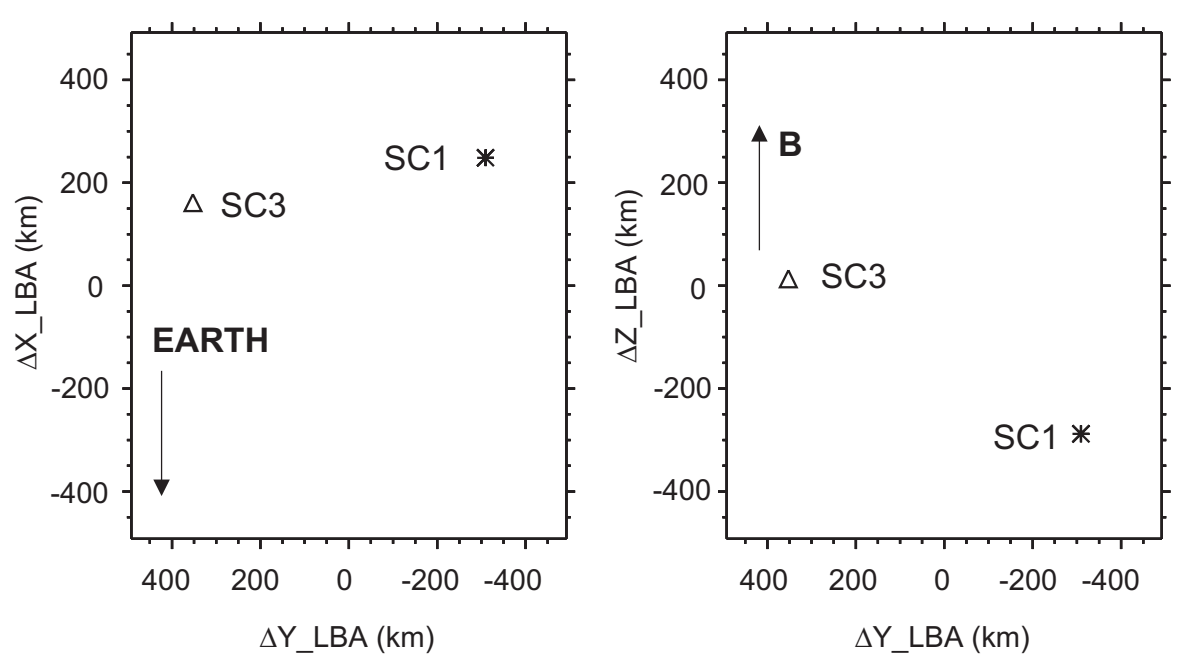

Fig. 6. Relative positions of Cluster 1 and 3 with respect to the center of mass of the four Cluster satellites in a local magnetic field-aligned coordinate system, showing that the two spacecraft do not lie along the same magnetic field line, but lie on separate field lines that are separated by about $650 \mathrm{~km}$. lieve that the IMF is in part controlling the generation of the structures. This is borne out by our observation that the frequency extent and amplitude of the structures increases as the angle of the IMF to the plasma flow direction (cone angle) decreases significantly from $90^{\circ}$. This dependence leads to the further conclusion that the bow shock is involved in some way in the generation of the structures, since it reacts to changing IMF and is known to be involved in the generation of electrostatic noise (solitons) that is convected downstream to form a turbulent region at the flanks of the Earth, outside the bow shock (Filbert and Kellogg, 1979).

Onsager et al. (1989) speculated that the broad-band, impulsive waves at frequencies greater than about $9 \mathrm{kHz}$ that are polarized parallel to the magnetic field and observed in the magnetosheath downstream from the shock by AMPTE were electron beam mode waves. As in the case of Coroniti et al. (1994), Onsager et al. (1989) did not have the waveforms available, but their criteria for these waves appear to fit very well the spectral representation of the bipolar pulses observed on Cluster in a similar region. Therefore, we believe that these are one and the same. Onsager et al. (1989) proposed three mechanisms for the generation of the electron beam required in order to produce the waves in the electron beam mode: acceleration by the cross-shock electric field, acceleration by lower hybrid frequency waves, and a magnetosheath "time of flight" mechanism similar to that in the Earth's foreshock (Filbert and Kellogg, 1979). In light of the recent findings that the broad-band waves are, in fact, potential structures, these mechanisms will need to be reexamined, but is beyond the scope of the present paper.

With regard to the low-frequency sinusoidal waves around $840-1120 \mathrm{~Hz}$ that are believed to be ion acoustic waves, it is possible that these waves are Doppler-shifted up in frequency. Since we do not have all of the necessary data at our disposal in order to determine this, we have used the parameters from a magnetosheath example taken from AMPTE data on 27 December 1984 contained in Onsager et al. (1989). The upstream parameters for this example are very similar to those of ACE for the Cluster data presented here and the spectral features of the broad-band waves observed by AMPTE are very similar to those of Cluster. Using the AMPTE parameters gives a maximum Doppler shift of $4 \mathrm{kHz}$. Since the ion plasma frequency is around 800 to $1000 \mathrm{~Hz}$ for the data shown in Fig. 2, we would expect Doppler-shifted ion acoustic waves no higher than $4 \mathrm{kHz}$. This is consistent with the low-frequency sinusoidal waves observed in Fig. 2, which are observed up to about $3 \mathrm{kHz}$. The solitary potential structures (bipolar pulses) are unlikely to be affected by a Doppler shift, because they are believed to be propagating at velocities on the order of a few thousand $\mathrm{km} / \mathrm{s}$, which is considerably higher than the magnetosheath velocity of $200-400 \mathrm{~km} / \mathrm{s}$. The low-frequency sinusoidal waves that are detected at the same time as the bipolar pulses may be important in understanding how the pulses are generated. One thing that is clear is that the detection of this lower frequency sinusoidal wave in the spin plane, which also contains the major component of the magnetic field, implies that this wave has a very measurable electric field component along the magnetic field and could thus, potentially provide a trapping mechanism for electrons.

The major conclusion to be derived from the Cluster WBD data obtained by two spacecraft in the dayside magnetosheath on 8 May 2001 is that the generation region must be extremely large. The fact that onsets of the bipolar pulses are observed almost at the same time on two spacecraft located about $750 \mathrm{~km}$ apart, and that the duration of the pulses and intensities of the pulses are similar and change similarly over time points most strongly to this conclusion. Whether the solitary potential structures that are observed in the magnetosheath are generated in the bow shock and propagate 
to each individual spacecraft along different magnetic field lines, or whether they are generated locally at each spacecraft in response to processes occurring at the bow shock is not currently known. We note, however, that although the separation of the two spacecraft is $750 \mathrm{~km}$, spacecraft 3 is only $153 \mathrm{~km}$ closer to the bow shock than spacecraft 1 . This would mean that if the structures were generated at the bow shock, they would only be travelling at $170 \mathrm{~km} / \mathrm{s}$ based on the delay of $0.9 \mathrm{~s}$ from spacecraft 3 to 1 , which is inconsistent with all other measurements of the velocities of this type of structure. Thus, it might be reasonable to assume that the structures are generated locally at each spacecraft in response to processes occurring at the bow shock, with the messengers of those processes travelling to each spacecraft at about $200 \mathrm{~km} / \mathrm{s}$.

\section{Summary}

The Cluster WBD instrument has obtained digital waveform measurements of the wave electric field sampled at $219.5 \mathrm{kHz}$ in the dayside magnetosheath from two spacecraft separated by about $750 \mathrm{~km}$. The waveforms show the presence of solitary bipolar pulses with time durations on the order of 25-100 $\mu \mathrm{s}$. These bipolar pulses are consistent with solitary waves observed in the magnetosheath and from other regions of space in which they are interpreted as solitary potential structures, and in this case, more specifically as electron phase space holes. There is no correlation between the individual structures from one spacecraft to another at these large distances. A spectrogram of these data covering a 21-minute time interval primarily shows broad-band features covering the range of $1 \mathrm{kHz}$ (the lower limit of the bandpass) up to $40 \mathrm{kHz}$, which is the typical representation of a pulse when using a windowed Fourier Transform.

The profiles of these broad-band features (onset, frequency extent, intensity, termination) when viewed over the 21-min interval are strikingly similar on the two Cluster spacecraft. This profile is also similar to that of the amplitude of the component of the magnetic field that lies in the spin plane of the spacecraft, the only plane that is sampled by WBD. This implies that the solitary potential structures associated with these broad-band features are propagating along the magnetic field, since they are not observed when the magnetic field lies primarily along the spin axis. The intensity and frequency extent of the broad-band features also appear to be dependent on the angle between the direction of the magnetic field and the direction of the plasma flow, with the intensity and frequency extent increasing as the angle decreases from $90^{\circ}$. Finally, and most important, the good correlation between the overall profile of the structures on one spacecraft and that on the other, when separated by $750 \mathrm{~km}$, implies that the generation region, whether local, at, or near the bow shock, is large.

Cluster WBD should be in a better position to observe these solitary potential structures in the magnetosheath, as well as in all other regions of its orbit, during the time period of 1 February through 30 May 2002. During this period, the interspacecraft separation distances will be on the order of $100-300 \mathrm{~km}$. We hope to be able to positively identify identical structures on more than one spacecraft, so that we can determine velocity and scale size. In addition, this will give us an opportunity to examine the all important issue of how the structures propagate, grow and decay. We also hope to be able to determine whether the structures coalesce early in their generation. With the aid of the potential control experiment, ASPOC, to shield photoelectrons from the spacecraft, we hope to be able to measure electrons to lower energies on the dayside than is possible without potential control. Electron and ion data, together with the WBD and other wave data, and electric and magnetic field data from multi-Cluster spacecraft, should provide us with the first opportunity to determine experimentally the generation mechanism, propagation, growth, and decay properties of these structures.

Acknowledgements. We acknowledge the support of NASA Goddard Space Flight Center through Contract NAS5-30730 and Grant NAG5-9947. Portions of the work were performed at the Jet Propulsion Laboratory, California Inst. of Tech. under contract with NASA. We acknowledge the ACE spacecraft investigation team for the use of their browse magnetic field and ion data. We thank the following individuals who were crucial in obtaining and processing the data: R. Huff, J. Dowell, R. Brechwald and I. Christopher of the University of Iowa, C. Abramo and A. Horton at DSN, K. Yearby and I. Willis of Sheffield University and M. Hapgood of Rutherford Appleton Laboratory. We also acknowledge use of some very valuable analysis tools supplied by GSFC, namely, SSCWeb and CDAWeb.

\section{References}

Anderson, R. R., Harvey, C. C., Hoppe, M. M., Tsurutani, B. T., Eastman, T. E., et al.: Plasma waves near the magnetopause, J. Geophys. Res., 87, 2087-2107, 1982.

Balogh, A., Dunlop, M. W., Cowley, S. W. H., Southwood, D. J., Thomlinson, J. G., et al.: The Cluster Magnetic Field Investigtaion, Space Sci. Rev., 79, 65-92, 1997.

Coroniti, F. V., Greenstadt, E. W., Moses, S. L., Tsurutani, B. T., and Smith, E. J.: On the Absence of Plasma Wave Emissions and the Magnetic Field Orientation in the Distant Magnetosheath, Geophys. Res. Lett., 21, 2761-2764, 1994.

Ergun, R. E., Carlson, C. W., McFadden, J. P., Mozer, F. S., Delory, G. T., et al.: FAST satellite observations of large amplitude solitary structures, Geophys. Res. Lett., 25, 2041-2044, 1998.

Filbert, P. C. and Kellogg, P. J.: Electrostatic Noise at the Plasma Frequency beyond the Earth's Bow Shock, J. Geophys. Res. 84, 1369-1381, 1979.

Franz, J. A., Kintner, P. M., and Pickett, J. S.: POLAR observations of coherent electric field structures, Geophys. Res. Lett., 25, 1277-1280, 1998.

Goldman, M. V., Oppenheim, M. M., and Newman, D. L. Nonlinear two-stream instabilities as an explanation for auroral bipolar wave structures, Geophys. Res. Lett., 13, 1821-1824, 1999.

Gurnett, D. A., Frank, L. A., and Lepping, R. P.: Plasma waves in the distant magnetotail, J. Geophys. Res., 81, 6059-6071, 1976.

Gurnett, D. A., Huff, R. L., and Kirchner, D. L.: The Wide-Band Plasma Wave Investigation, Space Sci. Rev., 79, 195-208, 1997. 
Kojima, H., Matsumoto, H., and Omura, Y.: Electrostatic solitary waves observed in the geomagnetic tail and other regions, Adv. Space Res., 23, 1689-1697, 1999a.

Kojima, H., Omura, Y., Matsumoto, H., Miyagut, K., and Mukai, T.: Characteristics of electrostatic solitary waves observed in the plasma sheet boundary: statistical analyses, Non. Proc. Geophys., 6, 179-186, 1999b.

Koskinen, H. E. J., Bostrom, R., and Holback, B.: Viking observations of solitary waves and weak double layers on auroral field lines, Phys. of Space Plasmas, SPI conference proceedings and reprints series, 7, 147-156, 1987.

Mangeney, A., Salem, C., Lacombe, C., Bougeret, J.-L., Perche, C., et al.: WIND observations of coherent electrostatic waves in the solar wind, Ann. Geophysicae, 17, 307-320, 1999.

Matsumoto, H., Kojima, H., Miyatake, T., Omura, Y., Okada, M., et al.: Electrostatic solitary waves (ESW) in the magnetotail: BEN wave forms observed by GEOTAIL, Geophys. Res. Lett., 21, 2915-1918, 1994.

Muschietti, L., Ergun, R. E., Roth, I., and Carlson, C. W.: Phasespace electron holes along magnetic field lines, Geophys. Res. Lett., 26, 1093-1096, 1999.

Newman, D. L., Goldman, M. V., Ergun, R. E., and Mangeney, A.:
Formation of double layers and electron holes in a current-driven space plasma, Phys. Rev. Lett., 87, 255 001-1-255 001-4, 2001.

Omura, Y., Kojima, H., Miki, N., and Matsumoto, H.: Twodimensional electrostatic solitary waves observed by Geotail in the magnetotail, Adv. Space Res., 24, 55-58, 1999.

Onsager, T. G., Holzworth, R. H., Koons, H. C., Bauer, O. H., Gurnett, D. A., Anderson, R. R., Luhr, H., and Carlson, C. W.: HighFrequency Electrostatic Waves Near Earth's Bow Shock, J. Geophys. Res., 94, 13 397-13 408, 1989.

Rodriguez, P.: Magnetosheath Electrostatic Turbulence, J. Geophys. Res., 84, 917-930, 1979.

Scarf, F. L., Frank, L. A., Ackerson, K. L., and Lepping, R. P.: Plasma wave turbulence at distance crossings of the plasma sheet boundaries and the neutral sheet, Geophys. Res. Lett., 1, 189192, 1974.

Singh, N.: Electron holes as a common feature of double-layerdrive plasma waves, Geophys. Res. Lett., 27, 927-930, 2000.

Temerin, M., Cerny, K., Lotko, W., and Mozer, F. S.: Observations of double layers and solitary waves in the auroral plasma, Phys. Rev. Lett., 48, 1175-1179, 1982.

Torrence, C. and Compo, G. P.: A practical guide to wavelet analysis, Bull. Amer. Meteor. Soc., 79, 61-79, 1998. 